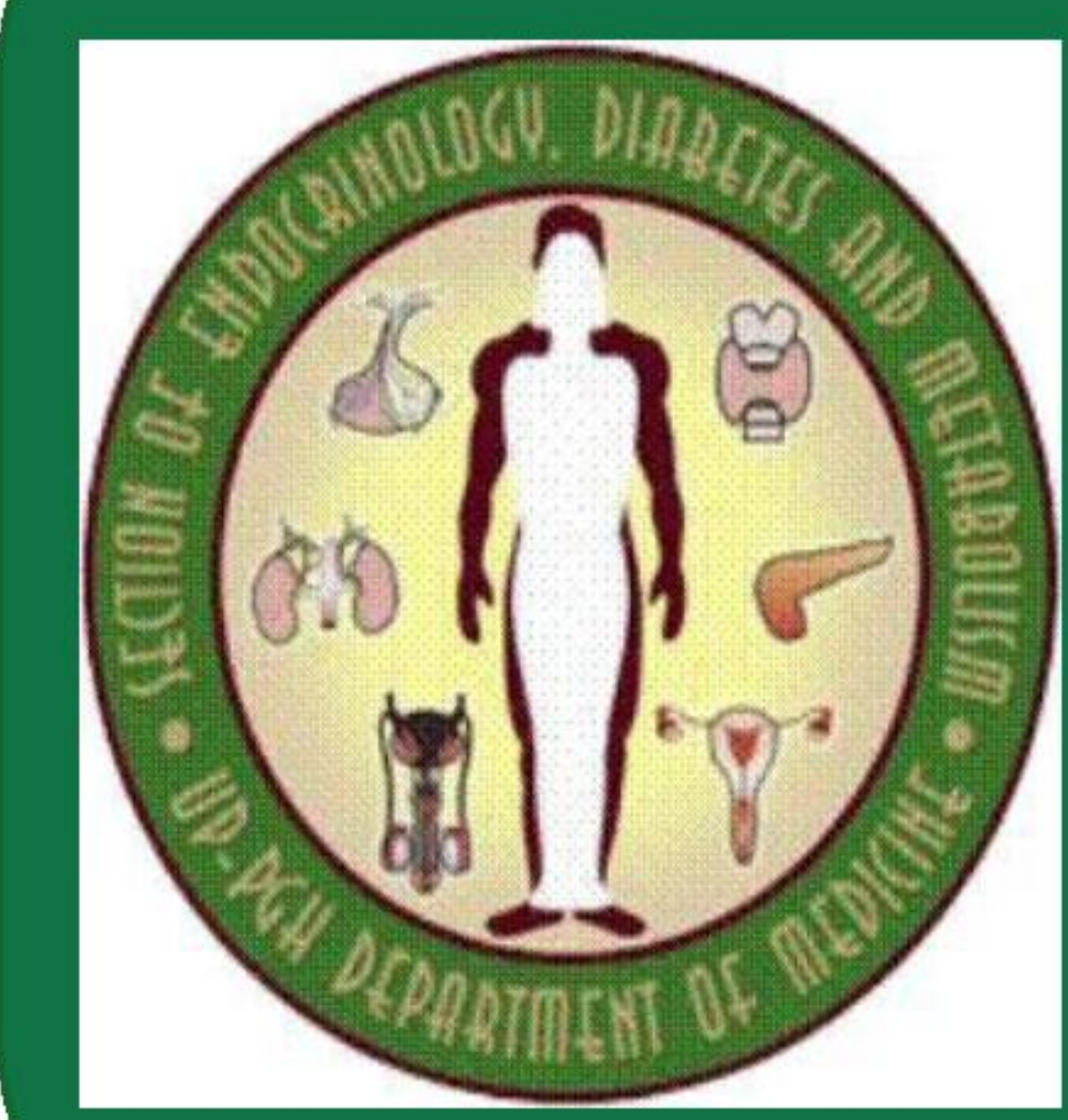

\title{
Tuberculous lymphadenitis mimicking noda metastasis in follicular variant papillary thyroid carcinoma
}

\author{
Marc Gregory Yu MD1, Jenny Maureen Atun MD²
}

1Section of Endocrinology, Diabetes, and Metabolism, Department of Medicine 2 Department of Pathology

University of the Philippines-Philippine General Hospital

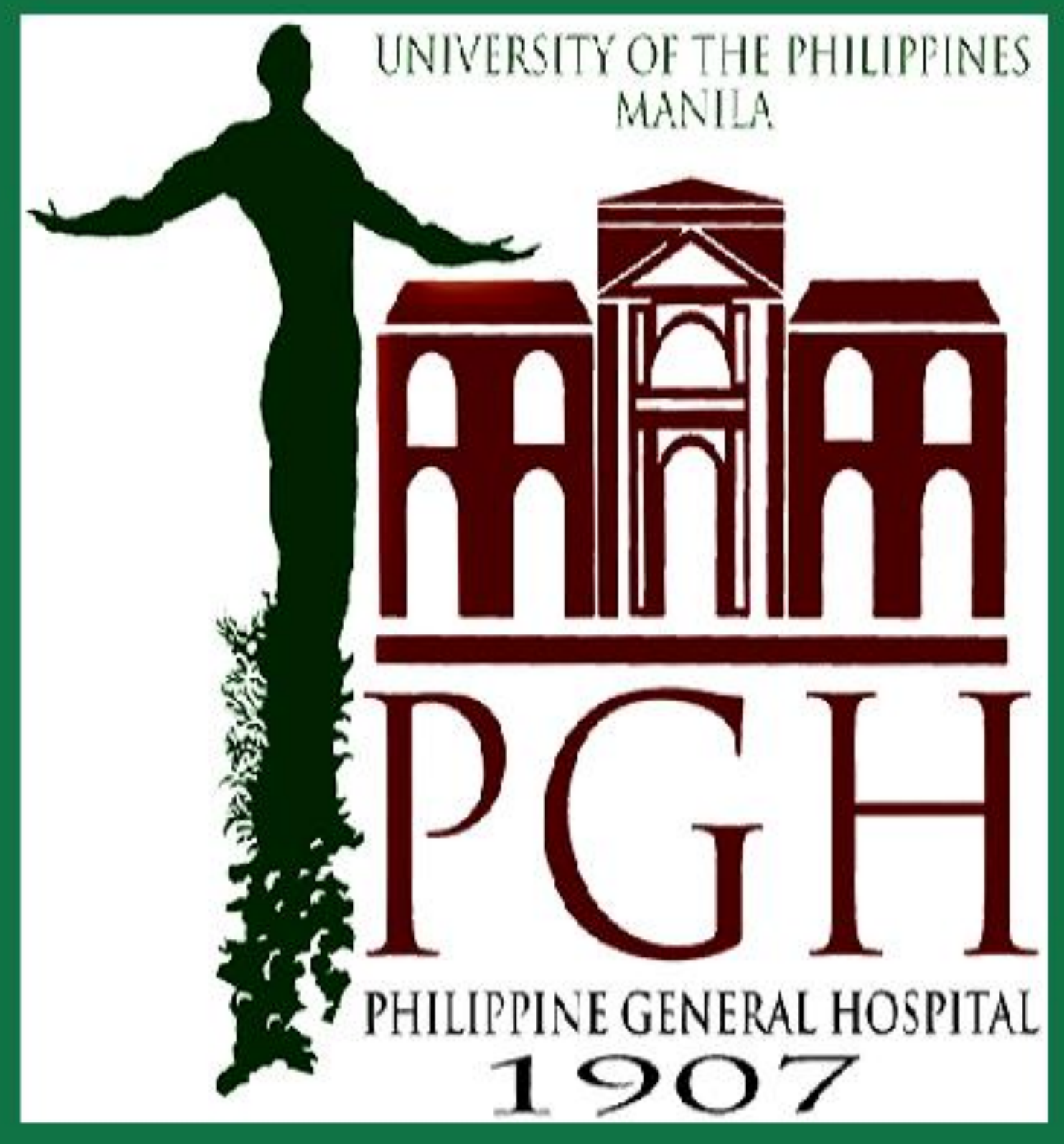
Manila, Philippines

\section{Background}

Tuberculous (TB) lymphadenitis is usually indistinguishable from lymph node metastasis due to PTC since the distribution and appearance of affected lymph nodes tends to be similar. In regions with high TB burden such as the Philippines, a proper preoperative evaluation is needed to distinguish these two entities and provide appropriate treatment.

\section{Objectives}

1. To present a case of TB lymphadenitis mimicking cervical node metastasis in a patient with follicular variant (FV)-PTC;

2. To discuss the radiologic and pathologic characteristics of both TB lymphadenitis and lymph node metastasis from PTC; and

3. To highlight the importance of a proper preoperative evaluation in the approach to PTC patients with lymphadenopathy.

\section{Case Presentation}

A 50-year-old Filipino presented at the outpatient clinic with a seven-year history of a gradually enlarging anterior neck mass. On physical examination, a firm, nontender mass measuring $5 \times 4 \times 2 \mathrm{~cm}$ was palpated at the left thyroid lobe with an associated $1 \times 1 \times 0.5 \mathrm{~cm}$ rubbery, nontender lymph node. The patient denied hypo- or hyperthyroid symptoms or compressive symptoms such as dyspnea, dysphagia, or odynophagia. He also denied chronic cough, night sweats, weight loss, or fever. There was no personal history of prior treatment or known exposure to TB, no previous head and neck irradiation, and no family history of thyroid disease. Blood tests revealed normal free thyroxine (fT4) levels at $21.3 \mathrm{pmol} / \mathrm{L}$ (N.V. 12-24 pmol/L) and thyroid stimulating hormone (TSH) levels at $0.59 \mathrm{ulU} / \mathrm{mL}$ (N.V. 0.3-3.8 ulU/mL.) A thyroid ultrasound (US) showed a $6.5 \times 5 \times 3 \mathrm{~cm}$ solid mass at the left lobe, plus a single $1.2 \times 1 \times 0.5 \mathrm{~cm}$ cervical lymph node with no suspicious features. Preoperative fine-needle aspiration biopsy (FNAB) of the thyroid mass revealed PTC. The patient underwent total thyroidectomy with neck dissection, with an unremarkable perioperative course. Histopathology confirmed FV-PTC, measuring $6.5 \mathrm{~cm}$ in widest dimension at the left lobe (Figure 1.) Histopathology of the lymph node, however, showed findings consistent with TB (Figure 2.) A chest X-ray plus two consecutive acidfast (AFB) stained sputum samples were negative for concomitant pulmonary TB. The patient started Category I anti-TB treatment and underwent adjuvant high-dose radioactive iodine therapy.

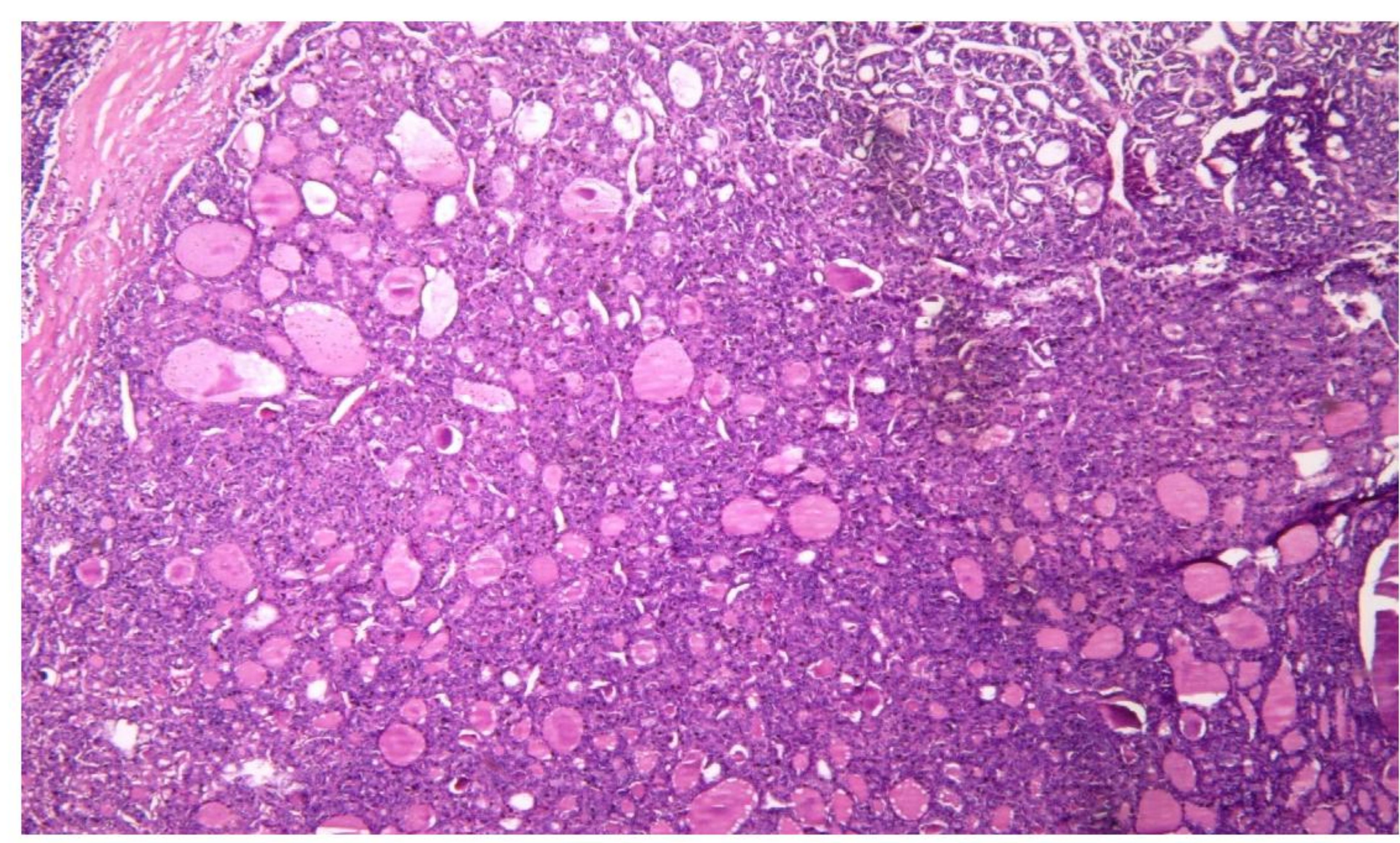

Figure 1. Scanning view of the thyroid mass showing tumor cells forming varied-sized follicles with densely eosinophilic colloid within the follicle lumen, consistent with FV-PTC. (Magnification: 40x)

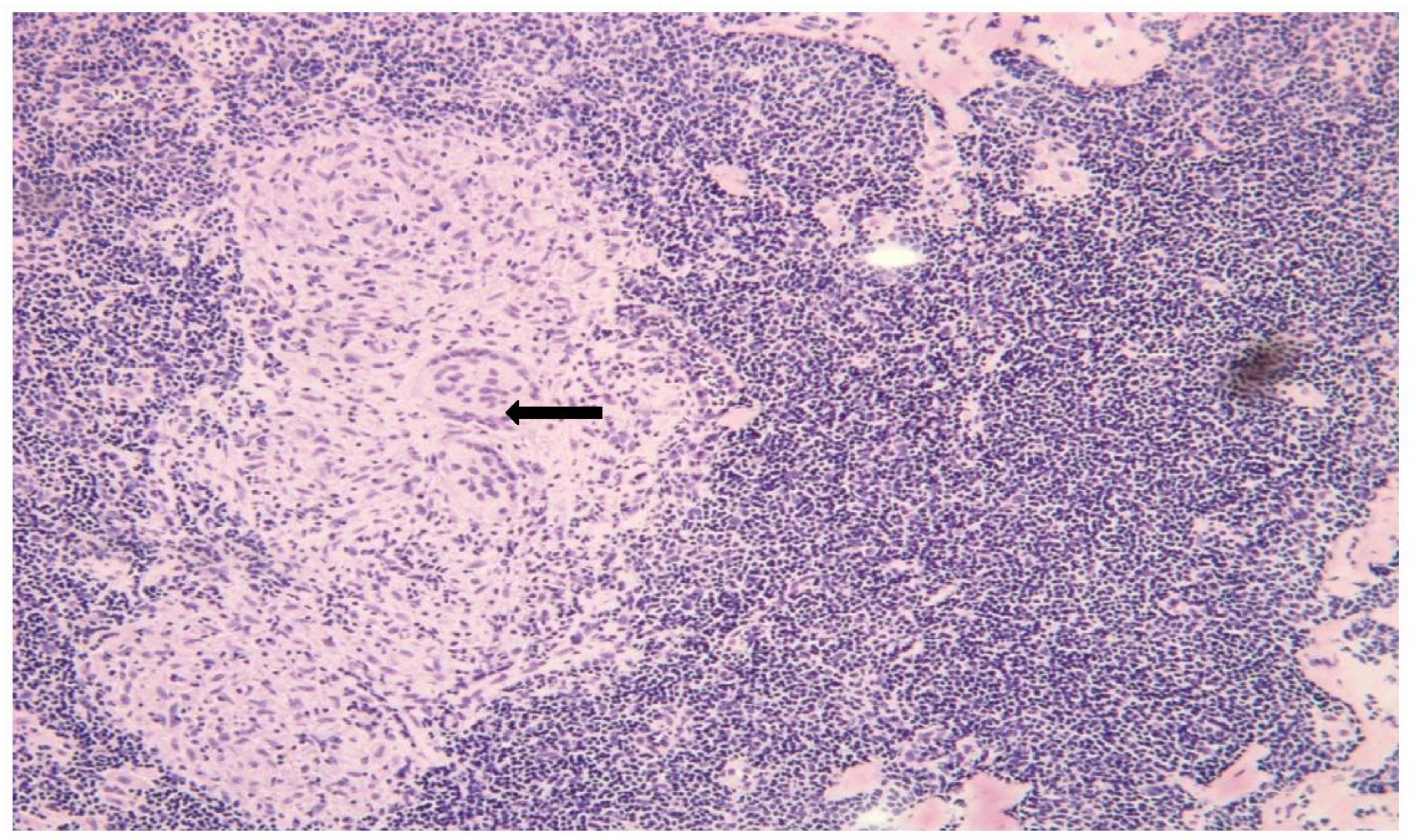

Figure 2. Low power view of the lymph node showing a granuloma composed of epithelioid histiocytes with abundant eosinophilic cytoplasm and multinucleated Langhans giant cells (black arrow), consistent with TB. (Magnification: 400x)

\section{Discussion}

Neck dissection is the standard of care in PTC patients with lymph node involvement. Although the procedure is relatively reliable and safe, it can still lead to post-operative complications and cosmetic problems. A careful preoperative evaluation is therefore paramount so that patients need not undergo an unwarranted neck dissection for other conditions.

Our patient initially presented with an anterior neck mass plus an associated cervical lymph node. Due to the documented malignancy, the proximity of the lymph node, and the absence of other clues suggesting another disorder, our primary consideration for the lymphadenopathy was metastasis. In this regard, US does not truly ascertain the etiology of lymphadenopathy as the distribution and sonographic appearance is virtually identical in TB adenitis and cervical node metastasis. Both preferentially occur in the supraclavicular area or posterior triangle of the neck, appearing as round and hypoechoic structures with frequent intranodal cystic necrosis and calcifications. Thus, without a high index of suspicion, the diagnosis of TB lymphadenitis can be easily missed.

Although a histopathologic examination remains the most accurate test for the diagnosis of TB adenitis, a preoperative FNAB can be done with the added option to run polymerase chain reaction (PCR) of the aspirate to further increase sensitivity and specificity. In retrospect, a prompt TB workup in our patient could have identified the disease preoperatively, leading to earlier initiation of antimycobacterial therapy and sparing the patient an unnecessary neck dissection.

Given the high TB prevalence in the region, it is interesting to note that this is the first documented case in the country and in Southeast Asia. This is also the first case involving FV-PTC, the most common PTC subtype. Our case is significant in the sense that current guidelines do not support the routine preoperative FNAB of unsuspicious lymph nodes in PTC, whose workup consists mainly of clinical and radiologic evaluation.

\section{Learning Points}

- Cervical lymphadenopathy in PTC is not always due to metastatic spread

- In endemic areas, TB lymphadenitis should be an important differential in a PTC patient with cervical lymphadenopathy.

- In developed countries, this differential is also valuable due to the increasing incidence of HIV/TB co-infection.

- When the index of suspicion is high, a proper preoperative TB workup is imperative to avoid an unwanted neck dissection. 